\title{
INFLUÊNCIA DA COMPOSIÇÃO DA RESINA TANINO-URÉIA-FORMALDEÍDO NAS PROPRIEDADES FÍSICAS E MECÂNICAS DE CHAPAS AGLOMERADAS ${ }^{1}$
}

\author{
Fabrício Gomes Gonçalves², Roberto Carlos Costa Lelis ${ }^{3}$ e José Tarcísio da Silva Oliveira ${ }^{4}$
}

\begin{abstract}
RESUMO - Na fabricação de aglomerados à base de madeira são utilizados adesivos sintéticos como melaminaformaldeído (MF), fenol-formaldeído (FF), uréia-formaldeído (UF), entre outros. Devido ao alto custo desses adesivos, pesquisas que visam a introdução de alterações nas suas formulações são importantes para a redução de custos no sistema produtivo. Desta forma, este trabalho teve como objetivo estudar a influência da adição de diferentes níveis de tanino de Acacia mearnsii em uma resina comercial à base de uréia-formaldeído nas propriedades físicas e mecânicas de chapas aglomeradas. As chapas aglomeradas foram confeccionadas com madeira de Eucalyptus urophylla. Foram avaliadas quatro composições de adesivo: T1 - 100\% de resina UF (testemunha); (T2) 90\% de resina UF $+10 \%$ de tanino 50\%; (T3) 85\% de resina UF $+15 \%$ de tanino 50\% e (T4) 70\% de resina UF + 30\% de tanino 50\%. Através dos ensaios, conclui-se que é possível adicionar tanino à resina uréia-formaldeído sem com isso prejudicar as propriedades físicas e mecânicas das chapas.
\end{abstract}

Palavras chaves: Aglomerado, Adesivo, Tanino e Eucalyptus urophylla.

\section{INFLUENCE OF THE COMPOSITION OF TANNIN-UREA-FORMALDEHYDE RESINS IN THE PHYSICAL AND MECHANICALS PROPERTIES OF PARTICLEBOARD}

\begin{abstract}
In the production of the wood base particleboards, synthetic adhesives are used as melaminaformaldehyde (MF), phenol-formaldehyde (FF), urea-formaldehyde (UF), among others. Due to the high cost of these adhesives, researches that a seek the introduction of alterations in their formulations are important for the reduction of costs in the productive system. This work had as objective verify the influence of the several levels of Acacia mearnsii tannin in a commercial resin of urea-formaldehyde an the physical and mechanical properties of the particleboard. The particleboard was produced with Eucalyptus urophylla wood. They were four treatments: T1 - 100\% of resin UF (control); (T2) $90 \%$ of the resin UF $+10 \%$ of the tannin $50 \%$; (T3) $85 \%$ of the resin UF $+15 \%$ of the tannin $50 \%$ and (T4) $70 \%$ of the resin UF $+30 \%$ of the tannin $50 \%$. The results showed that it is possible to add tannin to the resin urea-formaldehyde without to alter the physical and mechanical properties of the boards.
\end{abstract}

Keywords: Particleboard, Adhesive, Tannin and Eucalyptus urophylla.

\footnotetext{
${ }^{1}$ Recebido em 13.03.2006 e aceito para publicação em 19.05.2008.

${ }^{2}$ Escola Agrotécnica Federal de São João Evangelista, EAFSJE, Sao Joao Evangelista-MG .E-mail: < fabricio@agronet.gov.br>.

${ }^{3}$ Departamento de Produtos Florestais da Universidade Federal Rural do Rio de Janeiro (UFRRJ), Seropedica-RJ. E-mail: $<$ r.lelis@uol.com.br $>$.

${ }^{4}$ Departamento de Engenharia Rural da Universidade Federal do Espírito Santo (UFES). E-mail: <jtsilva@npd.ufes.br>
} 


\section{INTRODUÇÃO}

Nas indústrias de compensados e aglomerados, as resinas tradicionalmente utilizadas são as fenólicas e uréicas, sendo estas últimas de baixa resistência à umidade (ROFFAEL, 1982), ambas obtidas pela condensação de formaldeído com fenol ou uréia, respectivamente. Chow (1983) menciona que estas substâncias, por serem oriundas de derivados de petróleo, elevam o custo do adesivo e conseqüentemente o custo total das chapas. Após a crise do petróleo, houve então, uma grande preocupação em substituir estes derivados petroquímicos na indústria de painéis de madeira (CHOW, 1983; PIZZI e MITTAL, 1994). Outro motivo para a substituição de adesivos comerciais por adesivos naturais é devido ao problema da emissão de substâncias tóxicas - formaldeído para a atmosfera, como apresentado por Margosian (1990).

Hilling et al. (2002) e Calegari et al. (2004) citam que devido à oscilação dos preços do petróleo e a fragilidade dos produtos sintéticos advindos de fontes de matéria-prima não-renováveis, a utilização de resinas à base de extratos de casca de espécies florestais tem sido alvo de inúmeras investigações.

Os polifenóis (taninos) obtidos da casca ou da madeira de algumas espécies florestais, a exemplo da Acacia mearnsii e do Schinopsis sp, apresentam grande potencial para substituir os adesivos sintéticos. No entanto, o tanino oriundo da casca da A.mearnsii, ainda é o mais utilizado e desponta como sendo o mais promissor. Indústrias na África já utilizam resinas a base de tanino na confecção de chapas aglomeradas. No Brasil, em especial no Rio Grande do Sul, também são produzidas e utilizadas resinas à base de taninoformaldeído para a produção de aglomerados (HILLIG et al., 2002).

De acordo com Hilling et al. (2002), os adesivos à base de tanino além de serem mais baratos que os adesivos à base de fenol e formol, possuem satisfatória resistência à água. Dependendo da natureza do tanino, o mesmo pode ser enquadrado em duas classificações, ou seja, os hidrolisáveis e os condensáveis (GONÇALVES et al., 2003).

Hergert (1989) e Pizzi e Mittal (1983) citados por Carneiro et al. (2001), mencionam que os polifenóis hidrolisáveis estão presentes em extratos de cascas e madeiras de alguns gêneros de espécies florestais, sendo constituídos de misturas de fenóis simples, tais como pirogalol e ácido elágico, além de açúcares, principalmente a glicose. Os taninos hidrolisáveis podem ser utilizados como substitutos parciais do fenol na produção de resinas e adesivos à base de fenolformaldeído. No entanto, apresentam comportamento químico semelhante aos fenóis, substituídos com baixa reatividade com formaldeído. Este comportamento químico, associado à limitada produção mundial, impede que eles sejam efetivamente utilizados para a produção de adesivos fenólicos.

Os taninos condensados são formados por unidades de flavonóides, flavan 3-ol (catequina) e flavan 3-4 diol (leucoantocianidina) em vários graus de condensação (Haslan, 1966, citado por Tostes, 2003). Os taninos condensados apresentam grande importância na fabricação de adesivos, sendo aqueles provenientes dos extratos da casca de Acácia negra e da madeira de Quebracho os mais importantes taninos condensados produzidos industrialmente. Os taninos condensados constituem mais de 95\% da produção mundial de taninos comerciais ultrapassando a marca de 350.000 t/ano (GUANGCHENG et al., 1991).

Kim et al. (2003) avaliaram as propriedades físicas e mecânicas de chapas aglomeradas confeccionadas com tanino obtido de pinus e acácia negra. Os resultados foram satisfatórios, sendo afirmado pelos autores que a temperatura da prensa e o tempo de prensagem são fatores limitantes na utilização dos tipos de adesivos naturais aplicados para as propriedades avaliadas.

Vital et al. (2004) avaliaram a eficiência de tanino sulfitado extraído de Eucalyptus grandis e E.pellita e adesivo comercial de uréia-formadeído na confecção de chapas de flocos de E. grandis e Pinus elliottii. Os autores citam que as propriedades das chapas fabricadas apenas com adesivo à base de tanino comportaram-se de forma satisfatória e que as chapas produzidas com adesivos de taninos da casca de $E$. grandis foram superiores àquelas fabricadas com adesivos de taninos da casca de E. pellita somente.

O objetivo principal deste trabalho foi estudar a influência da adição de diferentes níveis de tanino de Acacia mearnsii em uma resina comercial à base de uréia-formaldeído nas propriedades físicas e mecânicas de chapas aglomeradas confeccionadas com a madeira de Eucalyptus urophylla. Uma vez que a madeira influencia as propriedades das chapas, foram 
objetivos também conhecer a densidade básica da madeira, bem como a porcentagem de extrativos na mesma.

\section{MATERIAIS E MÉTODOS}

\subsection{Determinação da densidade básica e preparo das partículas}

Foram coletadas duas árvores de Eucalyptus urophylla, com idade de 7 anos, em plantio localizado no campus da Universidade Federal Rural do Rio de Janeiro - UFRRJ, no município de Seropédica, Estado do Rio de Janeiro. Foram retirados discos de 2,5cm de espessura média, livres de nós e outros defeitos partindo da base e a 25, 50, 75 e 100\% da altura comercial visando a determinação da densidade básica média.

Os discos de madeira foram imersos em água, de forma que os mesmos mantivessem a umidade acima do ponto de saturação das fibras. Estes foram fracionados no sentido radial formando-se uma cunha. Em seguida procedeu-se a determinação do volume através do método de variação do peso do líquido em balança analítica (VITAL, 1984). As cunhas foram secas em estufa a $10 \pm 3$ ${ }^{\circ} \mathrm{C}$ até atingirem massa constante, com variação mínima de 0,05\% em massa, obtendo-se assim a massa seca.

As árvores foram transformadas em toretes e estes foram reduzidos manualmente a cavacos e posteriormente a partículas em um moinho de martelo. As partículas foram peneiradas em sistema vibratório e utilizadas àquelas que passaram pela peneira de $4,37 \mathrm{~mm}$ e ficaram retidas na peneira de 0,61mm. Estas partículas foram secas ao ar até atingirem uma umidade próxima de $16 \%$ (pesou-se amostras diariamente, obtendo-se a umidade por diferença após secagem em estufa a $103 \pm 3^{\circ} \mathrm{C}$ ) e posteriormente armazenada em sacos plásticos.

\subsection{Determinação dos teores de extrativos}

O teor de extrativo em água quente foi realizado de acordo com o descrito em Lelis (1995) mediante a utilização de 200ml de água destilada e 2 gramas de madeira em partículas (base seca). O material foi colocado em um balão de fundo chato de $250 \mathrm{ml}$, sendo o balão aquecido por 2 horas sob refluxo. A seguir, o material foi filtrado em cadinho de vidro sinterizado, de porosidade $\mathrm{n}^{\circ}$ 03, e lavado com 300ml de água quente sob vácuo, sendo em seguida secado em estufa a $103 \pm 3^{\circ} \mathrm{C}$ durante 24 horas e posteriormente pesado. A diferença entre a massa seca das partículas antes e após a extração, forneceu o teor de extrativo.
O teor de extrativo em água fria da amostra foi realizado baseado no descrito em Lelis (1995), mediante a utilização de $200 \mathrm{ml}$ de água destilada e 2 gramas de madeira em partículas (base seca). A mistura foi colocada em um erlenmeyer de $250 \mathrm{ml}$ submetendo-o à constante homogeneização por um período de 48 horas. Após este período, o material foi lavado com $300 \mathrm{ml}$ de água fria sob vácuo, sendo em seguida levado à estufa a $103 \pm 3^{\circ} \mathrm{C}$ durante 24 horas e pesado em seguida. A diferença entre a massa seca das partículas antes da extração e após a extração, forneceu o teor de extrativo.

A determinação do teor de extrativo em etanol/ ciclohexano foi realizada com base no descrito por Fengel e Przyklenk (1983). Foi utilizado o aparelho de Soxhlet mediante a utilização de 6 gramas de madeira em partículas (base seca) e 200ml de solvente etanol/cicloexano à uma proporção 1:2. As partículas foram colocadas em um recipiente confeccionado com papel de filtro e foram extraídas por um período de seis horas. O balão contendo os extrativos e solvente foi levado à um evaporador sob vácuo e após a recuperação do solvente, o balão com extrativo foi seco em um dessecador sob vácuo por um período de 24 horas. Da diferença entre a massa do balão seco antes e depois da extração, obteve-se a quantidade de extrativos em gramas e considerandose a quantidade de madeira em partículas (base seca) empregada, calculou-se o teor de extrativos em percentagem.

\subsection{Caracterização do adesivo}

O adesivo sintético utilizado foi o CASCAMITE PL 117 à base de uréia-formaldeído com alto teor de formol livre, doado pela empresa BORDEN QUÍMICA de São Paulo, SP. O adesivo PL 117 sofreu modificações através de substituição de parte do adesivo por tanino em pó, diluído com água destilada (50\%) e homogeneizado até o ponto em que não se observava a presença do pó no recipiente. O tanino foi doado pela empresa TANAC, de Montenegro, RS, sendo que o adesivo UF foi substituído nas proporções de 10\%, 15\% e 30\%.

A densidade dos adesivos modificados e da testemunha, foi determinada mediante a pesagem de 20,0 $\mathrm{ml}$ do adesivo, sendo este valor dividido pelo volume conhecido, obtendo-se o valor em $\mathrm{g} / \mathrm{ml}$.

Para o adesivo UF e suas modificações com tanino foram determinados o teor de sólidos, tempo de formação de gel e viscosidade segundo Tostes e Lelis (2001).

R. Árvore, Viçosa-MG, v.32, n.4, p.715-722, 2008 


\subsection{Preparo das chapas}

As formulações do adesivo foram aplicadas às partículas, no interior de um tambor giratório por meio de aspersão utilizando uma pistola pneumática, visando a completa homogeneização e envolvimento do adesivo nas partículas.

Através da utilização de uma caixa de madeira sem fundo, com dimensões de $40 \mathrm{~cm}$ de lado e $30 \mathrm{~cm}$ de altura, procedeu-se uma pré-prensagem, formando um colchão, o qual foi acondicionado entre duas lâminas de alumínio, espaçadas com duas barras de ferro, medindo 1,27 cm de espessura, entre as duas lâminas.

A prensagem dos colchões obedeceu às seguintes condições:

- Dimensões das chapas: 40,0 x 40,0 x 1,27cm;

- Densidade nominal das chapas: 0,60g/ $/ \mathrm{cm}^{3}$;

- Conteúdo de adesivo: 10\% sobre a massa de partículas (base seca);

- Temperatura de prensagem: $170^{\circ} \mathrm{C}$;

- Pressão de prensagem: 30kgf/cm²;

- Tempo de fechamento da prensa: 20seg.;

- Tempo de prensagem: 9min;

- Teor de umidade das partículas: 16\%;

- Endurecedor: 2\% de uma solução de $\left(\mathrm{NH}_{4}\right)_{2} \mathrm{SO}_{4}$ a $15 \%$ sobre o total de sólidos.

Após a prensagem, as chapas foram mantidas em sala climatizada por 21 dias à temperatura de $20^{\circ} \mathrm{C} \pm$ $1^{\circ} \mathrm{C}$ e umidade relativa de $65 \% \pm 5 \%$. A seguir, as bordas das chapas foram aparadas para posterior recorte dos corpos-de-prova para os diferentes ensaios, obedecendo ao disposto na norma ASTM D 1037 (ASTM, 1988).

\subsection{Ensaios físicos-mecânicos}

Além da determinação da densidade das chapas, foram realizados os seguintes ensaios físicos-mecânicos:

- Inchamento em Espessura após 2 h (IE2) e 24 h (IE24) de imersão em água fria;

- Resistência à flexão estática (Módulo de Ruptura - MOR e Módulo de Elasticidade - MOE);

- Tração perpendicular ao plano das chapas (Ligação Interna - LI).
Os ensaios foram realizados no Laboratório de Tecnologia da Madeira, do Departamento de Produtos Florestais da UFRRJ, sendo os ensaios mecânicos realizados em uma máquina universal de ensaio com capacidade de 10 toneladas.

\subsection{Análise dos dados}

O delineamento estatístico utilizado foi o inteiramente casualizado, procedendo-se a análise de variância (ANOVA) para todos os ensaios seguidos pelo teste de Duncan ao nível de 95\% de probabilidade, para comparação de médias entre os tratamentos, todas as vezes que a hipótese da nulidade fosse rejeitada. Para cada um dos tratamentos abaixo, procedeu-se a confecção de 5 chapas ( 5 repetições):

- T1 - 100\% de resina UF (testemunha);

- $\quad$ T2 - 90\% de resina UF + 10\% de tanino 50\%;

- Т3 - 85\% de resina UF + 15\% de tanino 50\%; e,

- $\mathrm{T} 4-70 \%$ de resina UF $+30 \%$ de tanino $50 \%$.

Os dados referentes ao Inchamento em Espessura após 2 e 24 horas de imersão em água foram transformados através da expressãoarcsen $\sqrt{x / 100}$, visando a homogeneidade na análise pelo teste de Duncan, os resultados estão apresentados em sua originalidade.

\section{RESULTADOS E DISCUSSÃO}

\subsection{Propriedades do adesivo UF e de suas modificações com tanino}

A Tabela 1 apresenta as características do adesivo comercial e suas modificações com o tanino para utilização na confecção das chapas.

A viscosidade é uma propriedade de grande importância, pois a mesma afeta diretamente a resistência na linha de cola dos adesivos (PIZZI e MITTAL, 1994). Nota-se que a adição de tanino ao adesivo UF acarretou de modo geral uma diminuição nos valores de viscosidade, sendo isso bastante positivo, do ponto de vista de aplicabilidade da cola, apesar de nem sempre a redução na viscosidade ser positiva, uma vez que há um limite de viscosidade em função da densidade da madeira trabalhada.

Com relação ao tempo de formação de gel, observase maiores valores com adição de tanino à resina UF. O tempo de formação de gel está ligado à acidez do 
adesivo uma vez que o $\mathrm{pH}$ interfere na reatividade do adesivo (TOSTES, 2003). Com adição de tanino à resina UF era de se esperar menores valores de tempo de formação de gel. Isso não aconteceu, provavelmente devido à reação dos taninos com o condensado UF, bloqueando, desta forma, os grupos metilol da resina e assim a polimerização foi, em parte, impedida. Segundo Pizzi (1979) há então uma copolimerização resultando condensados de flavonóide-UF-flavonóide.

\subsection{Densidade da madeira, teor de extrativos e propriedades das chapas}

A madeira de Eucalyptus urophylla apresentou densidade básica média de $0,57 \mathrm{~g} / \mathrm{cm}^{3}$ podendo ser classificada como sendo de densidade mediana.

Os teores de extrativos da madeira de Eucalyptus urophylla solúveis em água quente, água fria e em etanol/ciclohexano foram, respectivamente 5,74; 2,30 e 4,45\%. De acordo com Thaines et al. (1996) a temperatura exerce uma pequena diferença na solubilidade dos monossacarídeos, amidos, fenóis simples e hemicelulose de baixo peso molecular. É possível que os teores de extrativos presentes na madeira, não exerceram influência no processo de colagem por ocasião da confecção das chapas, uma vez que os valores foram baixos.

A Tabela 2 apresenta os valores médios encontrados para a Densidade dos painéis, Inchamento em espessura após 2 horas e 24 horas em água fria, Módulo de Ruptura, Módulo de Elasticidade e Ligação Interna para chapas da classe I.

A densidade das chapas de partículas é controlada por dois fatores principais. De acordo com Kelly (1977) citado por Peixoto e Brito (2000), estes dois fatores são a densidade da madeira e a razão de compactação da chapa. Esta razão de compactação é entendida como sendo a divisão da densidade da chapa pela densidade da madeira. Os resultados demonstraram que não houve diferença significativa entre os valores da densidade para os diferentes tratamentos, evidenciando a homogeneização das chapas fabricadas.

De forma geral, todas as propriedades físicomecânicas das chapas, em maior ou menor intensidade, são afetadas pela razão de compactação. Hilling et al. (2002) citam que taxas de compactação muito baixas não produzem bom contato entre as partículas, prejudicando a colagem e a resistência da chapa.

Hilling et al. (2002) mencionam que a maioria das densidades de chapas de partículas situam-se entre 0,4 e $0,8 \mathrm{~g} / \mathrm{cm}^{3}$. Neste estudo, as chapas produzidas apresentaram baixos coeficientes de variação, com o menor valor de densidade média de $0,59 \mathrm{~g} / \mathrm{cm}^{3}$ e o maior de $0,63 \mathrm{~g} / \mathrm{cm}^{3}$.

Apesar da ANOVA não detectar diferença estatística para o Módulo de Elasticidade (MOE) nos tratamentos, pode-se visualizar que com o aumento da adição do teor de tanino, houve um acréscimo nos valores médios absolutos do MOE. Mesmo com a máxima adição de tanino ao adesivo comercial, as chapas obtiveram o mesmo comportamento, além de torná-las mais resistentes.

Para o Módulo de Ruptura (MOR), aANOVAmostrou que não ocorreu diferença entre os tratamentos, embora houvesse uma tendência de aumento dos valores médios absolutos à medida que se acrescentou tanino à resina. O coeficiente de variação para MOR foi relativamente alto para os tratamentos, o que pode explicar a inexistência da diferença estatística, conjuntamente com o desvio padrão das amostras, também elevados. A composição de chapas de flocos com taninos extraídos da casca de Eucalyptus grandis e Eucalyptus pellita foi avaliado por Carneiro et al. (2004). Os autores afirmam que a combinação dos adesivos tânicos de eucalipto com os adesivos à base de uréia-formaldeído ocasionou aumento em algumas das propriedades mecânicas estudadas.

Tabela 1 - Valores médios para densidade, teor de sólidos, tempo de formação de gel e viscosidade do adesivo Uréia-Formaldeído (UF) e de suas modificações com o tanino de Acacia mearnsii

Table 1 - Results of the density, solid content, viscosity and gel time of adhesive UF and UF modified with change from tannin of Acacia mearnsii

\begin{tabular}{|c|c|c|c|c|}
\hline $\begin{array}{c}\text { Tratamentos } \\
\qquad(\mathrm{g} / \mathrm{ml})\end{array}$ & $\begin{array}{c}\text { Densidade } \\
\text { Sólidos (\%) }\end{array}$ & $\begin{array}{c}\text { Teor de } \\
\text { formação de gel }\end{array}$ & $\begin{array}{l}\text { Tempo de } \\
\text { (mPas) }\end{array}$ & Viscosidade \\
\hline T1 - 100\% de resina (testemunha) & 1,37 & 63,90 & $1 ’ 15^{\prime \prime}$ & 705 \\
\hline $\mathrm{T} 2-90 \%$ de resina $+10 \%$ de tanino $50 \%$ & 1,17 & 62,88 & 1'49', & 501 \\
\hline T3 - 85\% de resina $+15 \%$ de tanino $50 \%$ & 1,35 & 60,86 & 2'05’’ & 539 \\
\hline $\mathrm{T} 4-70 \%$ de resina $+30 \%$ de tanino $50 \%$ & 1,14 & 58,16 & $2^{\prime} 50^{\prime \prime}$ & 698 \\
\hline
\end{tabular}


Tabela 2 - Valores médios encontrados para as propriedades físicas e mecânicas das chapas de partículas Table 2 - Results of physical and mechanical properties of the particleboards

\begin{tabular}{|c|c|c|c|c|}
\hline \multirow[t]{2}{*}{ Propriedades avaliadas } & \multicolumn{4}{|c|}{ Tratamentos } \\
\hline & $\begin{array}{c}\mathrm{T} 1 \\
\text { 100\% de resina } \\
\text { (testemunha) }\end{array}$ & $\begin{array}{c}\mathrm{T} 2 \\
90 \% \text { de resina }+10 \% \\
\text { de tanino } 50 \% \\
\end{array}$ & $\begin{array}{c}\text { T3 } \\
85 \% \text { de resina } \\
+15 \% \text { de tanino } 50 \% \\
\end{array}$ & $\begin{array}{c}\mathrm{T} 4 \\
70 \% \text { de resina }+30 \% \\
\text { de tanino } 50 \% \\
\end{array}$ \\
\hline Densidade Aparente $\left(\mathrm{g} / \mathrm{cm}^{3}\right)$ & $\begin{array}{l}0,59 * \mathrm{~ns} \\
(10,17) \\
\end{array}$ & $\begin{array}{l}0,60 \mathrm{~ns} \\
(11,66) \\
\end{array}$ & $\begin{array}{l}0,59 \mathrm{~ns} \\
(10,91) \\
\end{array}$ & $\begin{array}{c}0,63 \mathrm{~ns} \\
(4,76) \\
\end{array}$ \\
\hline IE 2 horas (\%) & $\begin{array}{c}20,14 \mathrm{a}^{* *} \\
(25,28)\end{array}$ & $\begin{array}{l}20,80 \text { a } \\
(10,91)\end{array}$ & $\begin{array}{l}17,29 \text { a } \\
(18,18)\end{array}$ & $\begin{array}{l}12,26 \mathrm{~b} \\
(26,33)\end{array}$ \\
\hline IE 24 horas (\%) & $\begin{array}{c}27,84 \text { a } \\
(6,07) \\
\end{array}$ & $\begin{array}{c}26,51 \text { a b } \\
(10,22)\end{array}$ & $\begin{array}{l}25,4 \mathrm{ab} \\
(11,93)\end{array}$ & $\begin{array}{l}23,36 \mathrm{~b} \\
(12,71)\end{array}$ \\
\hline MOR (MPa) & $\begin{array}{c}13,53 \mathrm{~ns} \\
(29,70)\end{array}$ & $\begin{array}{c}12,94 \mathrm{~ns} \\
(35,56)\end{array}$ & $\begin{array}{c}14,12 \mathrm{~ns} \\
(18,44)\end{array}$ & $\begin{array}{c}15,89 \mathrm{~ns} \\
(16,87)\end{array}$ \\
\hline MOE (Mpa) & $\begin{array}{c}1.887 \mathrm{~ns} \\
(29,06)\end{array}$ & $\begin{array}{c}1.815 \mathrm{~ns} \\
(26,36)\end{array}$ & $\begin{array}{l}1.959 \mathrm{~ns} \\
(18,89)\end{array}$ & $\begin{array}{c}2.224 \mathrm{~ns} \\
(10,30)\end{array}$ \\
\hline LI (Mpa) & $\begin{array}{l}0,6874 \mathrm{~ns} \\
(40,68)\end{array}$ & $\begin{array}{c}0,6482 \mathrm{~ns} \\
(36,31)\end{array}$ & $\begin{array}{c}0,5698 \mathrm{~ns} \\
(26,83)\end{array}$ & $\begin{array}{c}0,5531 \mathrm{~ns} \\
(14,53)\end{array}$ \\
\hline
\end{tabular}

* Valor entre parênteses abaixo do ponto médio indica o Coeficiente de Variação (\%)

** Letras iguais na mesma linha não diferem estatisticamente entre si, pelo teste de Duncan, a 5\% de probabilidade; ns - não significativo ao nível de $95 \%$ de significância

Salienta-se que os valores encontrados são aceitáveis para a Classe I de aglomerados de acordo com a norma americana CS 236-66(ASTM, 1988), que exige um mínimo de $56 \mathrm{kgf} / \mathrm{cm}^{2}$ para MOR e $10.500 \mathrm{kgf} / \mathrm{cm}^{2}$ para MOE.

A adesão entre as partículas de madeira formadoras do aglomerado é medida pela propriedade de Ligação Interna. Para as chapas fabricadas com adesivo comercial modificadas pelo tanino, os valores de Ligação Interna foram, em geral, inferiores ao da testemunha.

Porém, neste ensaio, não ocorreu diferença estatística entre os tratamentos, apesar dos valores médios absolutos encontrados terem diminuído à medida que se acrescentou tanino. A bibliografia mostra que o tanino, por apresentar açúcares e gomas de elevado peso molecular, pode reduzir a resistência à linha de cola, provavelmente devido às poucas ligações existentes entre o tanino e o formaldeído (Pizzi e Mital, 1994 citados por Carneiro et al., 2001). Cabe ressaltar que os dados para todos os quatro tratamentos apresentaram um baixo desvio padrão, com altos coeficientes de variação, indicando poder haver pequenas áreas de baixa eficiência do adesivo ao longo da chapa.

Mesmo com a adição de tanino ao adesivo comercial, as chapas produzidas apresentaram valores superiores ao mínimo exigido pela norma americana CS 236-66 (ASTM, 1988) que é de $1,4 \mathrm{Kgf} / \mathrm{cm}^{2}$.

Para o Inchamento após 2 horas houve um decréscimo acentuado nos valores de Inchamento após
2 horas quando se substituiu 30\% da resina UF pela solução de tanino 50\% (T4), ocorrendo diferença significativa entre esse tratamento e os demais.

A adição de $10 \%$ e $15 \%$ de tanino à resina UF não alterou significativamente os valores de inchamento em espessura após 2 horas de imersão em água. O mesmo comportamento foi obtido para os tratamentos após 24 horas de imersão em água.

Após 24 horas de imersão em água, o tratamento T4 (70\% UF, 30\% de tanino) apresentou o menor valor de Inchamento em Espessura, sendo significativo somente com a testemunha (UF pura).

Apesar do tanino possuir açúcares em sua composição que poderia prejudicar a linha de cola, podendo ainda comprometer a resistência final do aglomerado (COPPENS et al, 1980), observou-se que tal fato não alterou as propriedades físicas das chapas. É importante frisar que o tanino empregado foi obtido industrialmente e que o mesmo sofreu provavelmente algum tratamento para retirada de açúcares indesejáveis no processo de colagem.

Deve-se acrescentar, entretanto, que todos os resultados obtidos com os adesivos comerciais modificados com tanino 50\% estão de acordo com o Inchamento máximo permitido para as chapas classificadas na Classe 1 da norma CS 236-66,que é de até $30 \%$. 
É importante frisar também que não foi empregado parafina na formulação dos adesivos, o que poderia reduzir ainda mais os valores de Inchamento em espessura.

Gonçalves et al. (2003) encontraram também valores de Inchamento em espessura abaixo de 30\% para chapas produzidas com UF modificada com $10 \%$ e $20 \%$ de tanino de Mimosa caesalpinifolia.

\section{CONCLUSÕES}

A adição de maior porcentagem de tanino ao adesivo comercial UF permitiu de forma significativa que a estabilidade dimensional das chapas fosse melhorada.

Nas condições em que o experimento foi realizado, os resultados mostraram que é possível adicionar tanino à resina uréia-formaldeído sem com isso prejudicar as propriedades físicas e mecânicas das chapas. Tanto para MOR, MOE, quanto para a LI, os valores encontrados estão dentro da especificação da norma americana CS 236-66 da ASTM para chapas de partículas e não diferiram estatisticamente daqueles obtidos em chapas de uréiaformaldeído.

\section{AGRADECIMENTOS}

Os autores agradecem às empresas BORDEN QUÍMICAe à TANAC S/Apelo fornecimento do adesivo Uréia-Formaldeído e tanino, respectivamente.

\section{REFERÊNCIAS BIBLIOGRÁFICAS}

\section{AMERICAN SOCIETY FOR TESTING AND} MATERIALS. Standard methods of evaluation of the properties of woodbase fiber and particle panel materials. Annual book of ASTM Standards, ASTM D 1037 - 78 B. Philadelphia, 1988.

CALEGARI, L. et al. Adição de aparas de papel reciclável na fabricação de chapas de madeira aglomerada. Revista Ciência Florestal, Santa Maria, RS, v. 14, n. 1, p. 193-204. 2004.

CARNEIRO, A. C. O. et al. Propriedades de chapas de flocos fabricadas com adesivo de uréia-formaldeído e de taninos da casca de Eucalyptus grandis W. Hill ex Maiden ou de Eucalyptus pellita F. Muell. Revista Árvore, v.28, n.5, p.715-724, 2004.

CARNEIRO, A. C. O. et al. Reatividade dos taninos da casca de Eucalyptus grandis para produção de adesivos. Revista Cerne, Lavras, MG, v.7, n.1, p. 1-9, 2001.
CHOW, S. Adhesive developments in Forest products. Wood Science and Technology. v. 17, p. 1-11, 1983.

COOPENS, H. A.; SANTANA, M. A. E.;

PASTORE, F. J. Tannin formaldehyde adhesive for exterior-grade plywood and particleboard Manufacture. Forest Products Journal. v.30, n. 4, p. 38-42, 1980.

FENGEL, D.; PRZYKLENK, M. Vergleichende Extraktbestimmungen zum Ersatz von Benzol durch Cyclohexan. Holz als Roh-und Werkstoff. v. 4, p. 193-194, 1983.

GONÇALVES, C. A. et al. Produção de chapas de madeira aglomerada com adesivo uréiaformaldeído modificado com tanino de Mimosa caesalpiniaefolia Bentham (sabiá). Revista Floresta e Ambiente, Seropédica, RJ, v. 10, n.1, p.18 - 26, jan./jul., 2003.

GUANGCHENG, Z., YUNLU, L.; YAZAKI, Y. Extractive yields, stiasny values and polyflavonoid contents in barks from six acacia species in Australia. Australian Forestry, v.54, p.154-156. 1991.

HILLING, E.; HASELEIN, C. R.; SANTINI, E. J. Propriedades mecânicas de chapas aglomeradas estruturais fabricadas com madeiras de pinus, eucalipto e Acácia-negra. Revista Ciência Florestal, Santa Maria, RS, v. 12, n. 1, p. 59-70, 2002.

KIM, S. et al. Physico-mechanical properties of particleboards bonded with pine and wattle tanninbased adhesives. Journal Adhesion Science Technology. V. 17, n. 14, p.1863-1875, 2003.

LELIS, R. C. C. Zur Bedeutung der

Kerninhaltsstoffe obligatorisch verkernter Nadelbaumarten bei der Herstellung von feuchtebeständigen und biologisch resistenten Holzspanplatten, am Beispiel der Douglasie (Pseudotsuga menziesii Mirb. Franco). 1995. 249f. Tese (Doutorado em Ciência Florestal) Forstliches Fakultät der Universität Göttingen, Göttingen, Alemanha, 1995.

MARGOSIAN, R. Initial fomaldehyde emission levels for particleboard manufactured in the United Satates. Forest Products Journal. (Technical note). v. 40, n. 6, p. 19-20, 1990.

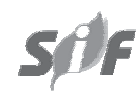

R. Árvore, Viçosa-MG, v.32, n.4, p.715-722, 2008 
PEIXOTO, G. L.; BRITO, E. O. Avaliação da granulometria de partículas de Pinus taeda combinadas com adesivos comerciais para a fabricação de aglomerados. Revista Floresta e Ambiente, Seropédica, RS, v. 7, n.1, p. 60 - 67, jan./dez, 2000.

PIZZI, A. The chemistry and development of tannin/urea-formaldehyde condensates for exterior wood adhesives. Journal of Applied polymer science, v. 23, p. 2777-2792, 1979.

PIZZI, A.; MITTAL, K. L. Handbook of Adhesive Technology, Marcel Dekker, Chapter 19, p. 347-358. 1994.

ROFFAEL, E. Die Formaldehydabgabe von Spanplatten und anderen Holzwerkstoffen. DRW-Verlag, Stuttgart, 154 p. 1982.

THAINES, F. et al. Composição química e características anatômicas da madeira de amescla, Trattinickia burseraefolia (MART.) Willd. SIMPÓSIO INTERNACIONAL SOBREECOSSISTEMAS FLORESTAIS, IV., 1996. Belo Horizonte. Anais... Belo Horizonte: BIOSFERA, 1996. p. 248-249.
TOSTES, A. S. Tanino da casca de Eucalyptus pellita (Muel) como fonte de adesivos para produção de chapas de madeira aglomerada. 2003. 85f. Dissertação (Mestrado em Ciências Ambientais e Florestais). Instituto de Florestas, Universidade Federal Rural do Rio de Janeiro, Seropédica, 2003.

TOSTES, A. S.; LELIS, R. C. C. Extrativos da casca de Eucalyptus pellita como fonte de adesivos para colagem de madeira. In: Simpósio Brasileiro de Pós-Graduação em Engenharia Florestal, I., 2001, Santa Maria, Anais... Santa Maria. CD ROM.

VITAL, B. R. Métodos de determinação da densidade da madeira. Sociedade de Investigações Florestais (SIF). Boletim Técnico, 1. Viçosa, MG. 21 p. 1984.

VITAL, B. R. et al. Adesivos à base de taninos das cascas de duas espécies de eucalipto para produção de chapas de flocos. Revista Árvore, Viçosa, MG, v. 28, n. 4, p. 571-582, 2004. 